\title{
Noncompact drift for relative equilibria and relative periodic orbits
}

\author{
Peter Ashwin * \\ Ian Melbourne ${ }^{\dagger}$ \\ Dept. of Math. and Comp. Sci. \\ University of Surrey \\ Guildford GU2 5XH, UK \\ Department of Mathematics \\ University of Houston \\ Houston, TX 77204-3476, USA
}

February 3, 1997

\begin{abstract}
In the context of equivariant dynamical systems with a compact Lie group $\Gamma$ of symmetries, Field and Krupa have given sharp upper bounds on the drifts associated with relative equilibria and relative periodic orbits. For relative equilibria consisting of points of trivial isotropy, the drifts correspond to tori in $\Gamma$. Generically, these are maximal tori. Analogous results hold when there is a nontrivial isotropy subgroup $\Sigma$, with $\Gamma$ replaced by $N(\Sigma) / \Sigma$.

In this paper, we generalize the results of Field and Krupa to noncompact Lie groups. The drifts now correspond to tori or lines (unbounded copies of $\mathbb{R})$ in $\Gamma$ and generically these are maximal tori or lines. Which of these drifts is preferred, compact or unbounded, depends on $\Gamma$ : there are examples where compact drift is preferred (Euclidean group in the plane), where unbounded drift is preferred (Euclidean group in three dimensional space) and where neither is preferred (Lorentz group).

Our results partially explain the quasiperiodic (Winfree) and linear (Barkley) meandering of spirals in the plane, as well as the drifting behavior of spiral bound pairs (Ermakova et al). In addition, we obtain predictions for the drifting of the scroll solutions (scroll waves and scroll rings, twisted and linked) considered by Winfree and Strogatz.
\end{abstract}

*Supported in part by EU HCM grant ERBCHBCT930503 at the CNRS-INSA 'Institut Non Linéaire de Nice', France

†Supported in part by NSF Grant DMS-9403624 and by ONR Grant N00014-94-1-0317 


\section{Introduction}

A standard feature of differential equations with symmetry is that equilibria occur in group orbits: if the point $x_{0}$ is an equilibrium, then every point on the group orbit through $x_{0}$ is an equilibrium. More generally, a relative equilibrium is any flow-invariant group orbit. This includes the case of a group orbit of equilibria and also includes rotating waves. A rotating wave is a flow-invariant group orbit with a periodic flow, the time-evolution corresponding to drift along the group orbit.

A normally hyperbolic relative equilibrium persists under small perturbations but the flow on the relative equilibrium need not persist. For example, a normally hyperbolic $\mathbf{S O}(2)$-orbit of equilibria typically perturbs to a rotating wave. This is a special case of the theory due to Field [9] (see also Krupa [14]) that we describe below.

The results of Field and Krupa are formulated under the assumption that the group of symmetries is compact. Barkley [3] observed that the curious meandering properties $[20,2]$ of spirals in spatially-extended chemical systems correspond to drifts along group orbits in the noncompact Euclidean group $\mathbf{E}(2)$. It is the purpose of this paper to extend the results of Field and Krupa on relative equilibria (and relative periodic orbits) to the case when the group of symmetries is a noncompact Lie group. In particular, we interpret certain aspects for the meandering of spirals within this context and arrive at many new predictions for the three dimensional analogue of spirals called scrolls [21].

Relative equilibria First, we summarize the relevant results of Field [9]. Suppose that $\Gamma \subset \mathbf{O}(n)$ is a compact Lie group acting on $\mathbb{R}^{n}$ and that $f: \mathbb{R}^{n} \rightarrow \mathbb{R}^{n}$ is a smooth $\Gamma$-equivariant vector field. Let $X=\Gamma x_{0}$ be a flow-invariant group orbit (so $\left.f\left(x_{0}\right) \in T_{x_{0}} X\right)$. We recall that the isotropy subgroup $\Sigma$ of $x_{0}$ is defined to be

$$
\Sigma=\left\{\gamma \in \Gamma: \gamma x_{0}=x_{0}\right\} .
$$

Suppose first, for simplicity, that $\Sigma=1$. Then

(a) The closure of the trajectory through $x_{0}$ is given by $K x_{0}$ where $K$ is a closed connected abelian subgroup of $\Gamma$ (a torus),

(b) The flow on $K x_{0}$ is an (irrational) linear torus flow with as many independent frequencies as the dimension of $K$.

(c) The relative equilibrium $\Gamma x_{0}$ is foliated by copies of $K x_{0}$.

(d) Generically, $\operatorname{dim} K$ is maximal. 
Recall that a torus in a compact Lie group $\Gamma$ is maximal if it is not contained in a torus of larger dimension. It is well-known that all maximal tori in $\Gamma$ are conjugate and therefore have the same dimension. This common dimension is called the rank of $\Gamma$. In this language, part of the above result of Field can be restated as follows: $\operatorname{rank}(\Gamma)$ is an upper bound for the number of independent frequencies in the flow on $K x_{0}$ and generically this bound is attained.

When $\Sigma \neq \mathbf{1}$, there is the following modification [9]. Let $N(\Sigma)$ denote the normalizer of $\Sigma$

$$
N(\Sigma)=\left\{\gamma \in \Gamma: \gamma \Sigma \gamma^{-1}=\Sigma\right\}
$$

Then the results are as before, with $\Gamma$ replaced by the quotient $N(\Sigma) / \Sigma$. In particular, generically $\operatorname{dim} K=\operatorname{rank}(N(\Sigma) / \Sigma)$.

Suppose now that $\Gamma$ is noncompact. (We also relax the assumption that $\Gamma$ acts on a finite dimensional space.) It follows from the structure theory of Lie groups that the maximal tori in a connected finite dimensional Lie group are conjugate. However, it is no longer true that every closed connected abelian subgroup of $\Gamma$ is a torus. This leads to the possibility of unbounded drifts. We describe our results for $\Gamma$ noncompact in the case $\Sigma=1$. (The case $\Sigma \neq 1$ is similar, again with $\Gamma$ replaced by $N(\Sigma) / \Sigma$.)

The analogue of part (a) above is that the subgroup $K$ is either a torus or a copy of $\mathbb{R}$. Part (b) is unchanged when $K$ is a torus. Similarly, (c) is unchanged. The main difference is part $(\mathrm{d})$. There are two upper bounds that must be taken collectively: generically it is the case that $K$ is either a maximal torus in $\Gamma$ or $K \cong \mathbb{R}$. Which of these possibilities is realized depends on $\Gamma$. For example, suppose that $\Gamma=\mathbf{S E}(n)$ (the orientation-preserving Euclidean transformations of $\mathbb{R}^{n}$ ). If $n$ is even, then generically $K$ is a maximal torus. If $n$ is odd, then generically $K \cong \mathbb{R}$. On the other hand, suppose that $\Gamma$ is the group of symplectic linear transformations in $\mathbb{R}^{2 n}$. Then, there is an open set of vector fields for which $K$ is a torus and also an open set of vector fields for which $K \cong \mathbb{R}$. When $\Gamma$ is a matrix group, we give a Lie-algebraic method for computing the likelihood that $K$ is a torus or $K \cong \mathbb{R}$.

Relative periodic orbits A flow-invariant $\Gamma$-invariant set $P$ is called a relative periodic orbit if the orbit space $P / \Gamma$ is an ordinary periodic orbit. When $\Gamma$ is compact, Krupa [14] shows that similar results to those for relative equilibria are valid, with maximal tori generalized to Cartan subgroups. Krupa's results are formulated for relative periodic orbits in the vicinity of a relative equilibrium. Field [10] removes this restriction. Again, we generalize these results to the case when $\Gamma$ is not compact.

The paper is organized as follows. In Section 2, we recall the relevant aspects of the theory of maximal tori for noncompact Lie groups. In particular, we show 
that a typical element of the Lie algebra generates a maximal torus or a copy of $\mathbb{R}$. The examples considered in Section 3 demonstrate that, subject to this restriction, anything is possible. The corresponding theory of Cartan subgroups for nonconnected Lie groups is described in Section 4.

In Section 5, we turn attention to infinite-dimensional dynamical systems with noncompact symmetry group. The results of Field [9] and Krupa [14] on relative equilibria and relative periodic orbits are extended in Sections 6 and 7 respectively. In particular, we show that the generic drift of relative equilibria and relative periodic orbits can be computed at the Lie-theoretic level. Applications to Euclideanequivariant problems are considered in Section 8. We recover certain aspects of the meandering of spirals and we make predictions for the meandering of scroll waves. Finally we discuss some effects introduced by the presence of additional $S^{1}$ symmetry in the equations.

\section{Maximal tori in noncompact Lie groups}

Let $G$ be a finite dimensional second countable Lie group. We do not assume that $G$ is compact. Let $L G$ denote the Lie algebra. Recall that each element $\xi \in L G$ is an infinitesimal generator for a one-parameter subgroup $\exp (t \xi), t \in \mathbb{R}$, contained in $G$. Let $K(\xi)$ denote the closure

$$
K(\xi)=\overline{\{\exp (t \xi), t \in \mathbb{R}\}} .
$$

Proposition 2.1 The subgroup $K(\xi) \subset G$ is isomorphic either to a torus $\mathbf{T}^{p}$ or to a line $\mathbb{R}$.

Proof The subgroup $K(\xi)$ is a Lie group (since it is closed) and it is connected and abelian. Therefore $K(\xi) \cong \mathbf{T}^{p} \times \mathbb{R}^{q}$ for some nonnegative integers $p$ and $q[5$, Chapter I, Theorem 3.6].

Let $\pi_{2}$ denote the projection $\pi_{2}: \mathbf{T}^{p} \times \mathbb{R}^{q} \rightarrow \mathbb{R}^{q}$. The map $h: \mathbb{R} \rightarrow \mathbb{R}^{q}$ defined by $h(t)=\pi_{2}(\exp t \xi)$ is a smooth homomorphism and has the form $h(t)=t v$ for some $v \in \mathbb{R}^{q}$. By construction, the image of $h$ is dense in $\mathbb{R}^{q}$ and it follows that $q=0$ or $q=1$.

If $v=0$, then $K(\xi) \subset \mathbf{T}^{p}$. If $v \neq 0$, then $q=1$ and $K(\xi)$ is an embedded submanifold of $\mathbf{T}^{p} \times \mathbb{R}$ spiraling up or down the cylinder with no accumulation points. Hence $K(\xi) \cong \mathbb{R}$.

Suppose now that $G$ is connected. Then there is a maximal compact subgroup $K \subset G$ and this subgroup is unique up to conjugacy, see for example [6]. Moreover $G$ is homeomorphic to $K \times \mathbb{R}^{n}$ for some $n$. Recall that the compact connected Lie group 
$K$ contains a maximal torus $\mathrm{T}^{d}$ which is itself unique up to conjugacy in $K$. It follows that any compact connected abelian subgroup of $G$ is conjugate to a subgroup of $\mathbf{T}^{d}$. In particular, we are justified in calling $\mathrm{T}^{d}$ a maximal torus of $G$ and in defining $\operatorname{rank}(G)=\operatorname{rank}(K)=d$.

Corollary 2.2 Let $G$ be a connected Lie group. Suppose that $\xi \in L G$ with associated subgroup $K(\xi) \subset G$. Either $K(\xi)$ is conjugate to a subgroup of the maximal torus $\mathbf{T}^{d}$ or $K(\xi)$ is isomorphic to a copy of $\mathbb{R}$.

Remark 2.3 Although the maximal torus $\mathrm{T}^{d}$ is unique up to conjugacy in $G$, there is no such uniqueness result for $\mathbb{R}$. Indeed, there are zero copies of $\mathbb{R}$ when $G$ is compact, a unique copy of $\mathbb{R}$ when $G=\mathbb{R}$ and uncountably many nonconjugate copies of $\mathbb{R}$ when $G=\mathbb{R}^{2}$. In Example 3.3 below, we give an example where there are precisely two copies of $\mathbb{R}$ in $G$.

It turns out that every noncompact connected Lie group contains at least one copy of $\mathbb{R}$. Since we could not find a proof (or even a statement) of this elementary result in the literature, we have given a proof in the appendix.

Although, Corollary 2.2 allows the possibility that $K(\xi)$ is conjugate to a proper subgroup of $\mathrm{T}^{d}$, this is an exceptional occurrence. We prove

Theorem 2.4 For almost every $\xi \in L G$, the subgroup $K(\xi)$ is conjugate either to the maximal torus $\mathbf{T}^{d}$ or to a copy of $\mathbb{R}$.

More precisely, define the set of special elements of $L G$ :

$$
S=\left\{\xi \in L G, K(\xi) \text { is conjugate to a proper subgroup of } \mathbf{T}^{d}\right\} .
$$

We prove that $S$ is of first category and has zero Lebesgue measure in $L G$.

First, we prove the corresponding result at the group level. For $g \in G$, we define the group generated by $g$

$$
H(g)=\overline{\left\{g^{n}, n \in \mathbb{Z}\right\}} .
$$

Lemma 2.5 For almost every $g \in G$, the subgroup $H(g)$ is conjugate either to the maximal torus $\mathrm{T}^{d}$ or to a noncompact group.

Proof We prove the 'almost every' statement first in the measure-theoretic sense and second in the Baire category sense.

Let $S$ denote the set of 'special' elements of $G$ : those elements that generate a group that is compact but not a maximal torus. Let $S_{0}=\mathrm{T}^{d} \cap S$. We use the fact 
that $S_{0}$ has measure zero in $\mathrm{T}^{d}$ to show that $S$ has Lebesgue measure zero in $G$. (To say that a set $S$ has Lebesgue measure zero in the $n$-dimensional manifold $G$ means that the image of any chart when restricted to $S$ has Lebesgue measure zero in $\mathbb{R}^{n}$.)

We follow closely the proof in [5, Chapter IV, Theorem 2.11]. The conjugation map $\rho: G / \mathbf{T}^{d} \times \mathbf{T}^{d} \rightarrow G$ defined by $\rho(g, \tau)=g \tau g^{-1}$ is a smooth map and hence maps sets of measure zero to sets of measure zero. (This would not be true if we replaced $G / \mathrm{T}^{d}$ by $G$ since the dimensions would be wrong.) Since $S_{0}$ has measure zero in $\mathbf{T}^{d}$, it follows that $G / \mathbf{T}^{d} \times S_{0}$ has measure zero in $G / \mathbf{T}^{d} \times \mathbf{T}^{d}$ and hence that $\rho\left(G / \mathbf{T}^{d} \times S_{0}\right)$ has measure zero in $G / \mathbf{T}^{d}$. It thus suffices to prove that $\rho\left(G / \mathbf{T}^{d} \times S_{0}\right)=S$.

Clearly $S$ is preserved by conjugation and it follows that $\rho\left(G / \mathbf{T}^{d} \times S_{0}\right) \subset S$. Conversely, suppose that $s \in S$. By definition, $H(s)$ is conjugate to a subgroup of the maximal compact subgroup $K$. By the theory of maximal tori, $H$ is conjugate to a subgroup of $\mathbf{T}^{d}$. Hence $s=\rho(g, \tau)$ for some $\tau \in \mathbf{T}^{d}$. Since $s$ does not generate a maximal torus, neither does $\tau$. Hence $s \in \rho\left(G / \mathrm{T}^{d} \times S_{0}\right)$ as required.

Next, we prove the Baire category version of the lemma. By Kronecker's Theorem [5, Chapter I, Theorem 4.13], $S_{0}=\cup X_{i}$ is a countable union of codimension one submanifolds of $\mathbf{T}^{d}$. Hence $S$ is a countable union of codimension one submanifolds $\rho\left(Y_{i}\right)$ where $Y_{i}=G / \mathbf{T}^{d} \times X_{i}$. It suffices to show that $\rho\left(Y_{i}\right)$ is of first category in $G$ for each $i$.

Consider the smooth map $\left.\rho\right|_{Y_{i}}: Y_{i} \rightarrow G$ of manifolds where $\operatorname{dim} Y_{i}<\operatorname{dim} G$. Every point in $Y_{i}$ is a critical point for $\left.\rho\right|_{Y_{i}}$ and hence the set $G-\rho\left(Y_{i}\right)$ coincides with the set of regular values of $\left.\rho\right|_{Y_{i}}$. By Sard's Theorem [1, Theorem E.4], this set is residual (it is here that we use the second countability of $G$ ) and hence $\rho\left(Y_{i}\right)$ is first category in $G$. (In fact Sard's Theorem implies also that $\rho\left(Y_{i}\right)$ has zero measure in $G$, but the first proof did not require Sard's Theorem or the precise characterization of $S_{0}$ provided by Kronecker's Theorem.)

Proof of Theorem 2.4 First, observe that $K(t \xi)=K(\xi)$ for all nonzero $t \in \mathbb{R}$. Hence, we may restrict without loss to $\xi \in V$ where $V$ is a neighborhood of the origin in $L G$ such that $\left.\exp \right|_{V}$ is a diffeomorphism.

It follows from Lemma 2.5 that the group $H(\exp \xi)$ is a maximal torus or noncompact for almost every $\xi \in V$. But $H(\exp \xi) \subset K(\xi)$ so we have that $K(\xi)$ contains a maximal torus or is noncompact. This fact, together with Corollary 2.2, implies that $K(\xi)$ is conjugate to a maximal torus or $\mathbb{R}$.

\section{$3 \quad$ Preference for the maximal torus or for $\mathbb{R}$}

In the previous section, we showed that $K(\xi) \cong \mathbf{T}^{d}$ or $K(\xi) \cong \mathbb{R}$ almost always. In this section, we discuss which of these possibilities might be preferred over the other. 
The answer depends very much on the group. As the examples below demonstrate, any of the following are possible (i) the maximal torus is preferred, (ii) the real line is preferred, (iii) neither is preferred.

Given a Lie group $G$ we have a partition of the Lie algebra

$$
L G=L_{T} G \cup L_{R} G,
$$

where

$$
L_{T} G=\{\xi \in L G: K(\xi) \text { is a torus }\}
$$

and

$$
L_{R} G=\{\xi \in L G: K(\xi) \cong \mathbb{R}\}
$$

Recall that $G$ is a matrix group if $G$ is isomorphic to a closed subgroup of $\mathbf{G L}\left(\mathbb{R}^{n}\right)$ for some $n$.

Lemma 3.1 Suppose that $G$ is a matrix group and that $\xi \in L G$. Then

(a) $K(\xi)$ is a torus if and only if $\xi$ is a semisimple matrix and all eigenvalues of $\xi$ have zero real part.

(b) $L_{T} G$ and $L_{R} G$ are semialgebraic subsets of $L G$.

Proof Since $G$ is a matrix group, the exponential map exp : $L G \rightarrow G$ is defined by the standard formula

$$
\exp \xi=\sum_{n=0}^{\infty} \frac{\xi^{n}}{n !}
$$

Without loss of generality, we may suppose that the matrix $\xi$ is in Jordan normal form. An easy calculation shows that $K(\xi)$ is compact if and only if $\xi$ is semisimple and all eigenvalues have zero real part. Part (b) follows from the fact that these are semialgebraic conditions on the matrix $\xi$. For example, to see that semisimplicity is semialgebraic, consider the set

$$
X=\left\{(\xi, v, \lambda) \in \mathbb{R}^{n^{2}} \times \mathbb{R}^{n} \times \mathbb{C}:(\xi-\lambda I) v \neq 0, \quad(\xi-\lambda I)^{2} v=0\right\} .
$$

Let $p: \mathbb{R}^{n^{2}} \times \mathbb{R}^{n} \times \mathbb{C} \rightarrow \mathbb{R}^{n^{2}}$ be projection. By the Tarski-Seidenberg Theorem [16], the image of the semialgebraic set $X$ under $p$ is semialgebraic and hence $Y=p(X) \cap L G$ is a semialgebraic subset of $L G$. But $Y$ is precisely the set of nonsemisimple matrices in $L G$. 
Lemma 3.1 shows that $L_{T} G$ is semialgebraic and therefore contains a stratum of minimum codimension. We define this to be the codimension of the event that $K(\xi)$ is a maximal torus, $\operatorname{codim}_{G} \mathbf{T}^{d}=\operatorname{codim} L_{T} G$ and write simply codim $\mathbf{T}^{d}$. Similarly, we define $\operatorname{codim} \mathbb{R}=\operatorname{codim}_{G} \mathbb{R}=\operatorname{codim} L_{R} G$. We suspect that this can be generalized to non-matrix groups.

Example 3.2 If $G$ is compact, then almost every $\xi \in L G$ generates a maximal torus in $G\left(\operatorname{codim} \mathbf{T}^{d}=0\right)$.

Example 3.3 If $G=\mathbb{R}^{n}$, each nonzero $\xi$ generates a copy of $\mathbb{R}\left(\operatorname{codim} \mathbf{T}^{0}=n\right.$, $\operatorname{codim} \mathbb{R}=0$ ). When $n \geq 2$, there are uncountably many distinct (nonconjugate) copies of $\mathbb{R}$. Of course, $\operatorname{rank}(G)=0$.

More generally, suppose that $G$ is the group of upper triangular real $n \times n$ matrices. Then $G$ is homeomorphic to $\mathbb{R}^{n(n+1) / 2}$ and $\operatorname{rank}(G)=0$. Each nonzero $\xi \in L G$ generates a line. The same is true for any closed subgroup of $G$.

An important special case is the two dimensional Lie group $G$ consisting of matrices

$$
\left(\begin{array}{cc}
a & b \\
0 & 1
\end{array}\right), \quad a, b \in \mathbb{R}, \quad a>0 .
$$

(This is the group of orientation-preserving affine transformations on the line, $x \mapsto$ $a x+b$.) Any element of $G$ is conjugate to an element with either $a=1$ or $b=0$. Hence, there are, up to conjugacy, precisely two copies of $\mathbb{R}$ in $G$ :

$$
\left(\begin{array}{cc}
e^{t} & 0 \\
0 & 1
\end{array}\right), \quad\left(\begin{array}{cc}
1 & t \\
0 & 1
\end{array}\right) .
$$

For almost every $\xi \in L G$, the subgroup $K(\xi)$ is conjugate to the first copy of $\mathbb{R}$, but the second copy of $\mathbb{R}$ occurs for a codimension one subspace of $L G$. Note that the two copies of $\mathbb{R}$ are quite distinct, the first arising from an eigenvalue with nonzero real part, the second arising from nonsemisimplicity.

These examples are extreme in the sense that in Example $3.2 K(\xi)$ is always compact, while in Example $3.3 K(\xi)$ is never compact except in the trivial case $\xi=0$. By way of contrast, we consider the groups $\operatorname{SE}(n)$ and $\operatorname{Sp}(2 n, \mathbb{R})$.

Example 3.4 Let $G=\mathbf{S E}(n)$, the Euclidean group of orientation preserving isometries of $\mathbb{R}^{n}$. Then $\mathbf{S E}(n)=\mathbf{S O}(n)+\mathbb{R}^{n}$ (semidirect product) and $d=\operatorname{rank}(G)=$ $[n / 2]$. There is a unique copy of $\mathbb{R}$ up to conjugacy. We show

When $n$ is even, $\operatorname{codim} \mathbf{T}^{d}=0$ and $\operatorname{codim} \mathbb{R}=1$. 
When $n$ is odd, $\operatorname{codim} \mathbf{T}^{d}=1$ and $\operatorname{codim} \mathbb{R}=0$.

The general element of $G=\mathbf{S E}(n)$ can be written as $(A, v)$ where $A \in \mathbf{S O}(n)$ and $v \in \mathbb{R}^{n}$ and is identified with the $(n+1) \times(n+1)$ matrix

$$
\left(\begin{array}{cc}
A & v \\
0 & 1
\end{array}\right) \text {. }
$$

Hence the Lie algebra $L G$ consists of matrices of the form

$$
\xi=\left(\begin{array}{cc}
B & w \\
0 & 0
\end{array}\right),
$$

where $B$ is a skew-symmetric $n \times n$ matrix and $w \in \mathbb{R}^{n}$. In particular, all eigenvalues of $B$, and hence $\xi$, have zero real part. It follows that $K(\xi)$ is compact if and only if $\xi$ is semisimple.

When $n$ is even, it is generically the case that the eigenvalues of $B$ are distinct and nonzero. Hence, $\xi$ is semisimple. In a one-parameter family, it can happen that $B$ has multiple or zero eigenvalues, in which case $\xi$ is nonsemisimple for generic choices of $w \in \mathbb{R}^{n}$.

When $n$ is odd, the skew-symmetric matrix $B$ is automatically singular. By a change of coordinates, we can arrange that the last two rows of $\xi$ have the form

$$
\left(\begin{array}{cccc}
0 & \cdots & 0 & w_{n} \\
0 & \cdots & 0 & 0
\end{array}\right),
$$

where $w_{n} \in \mathbb{R}$. It follows that generically $\xi$ is nonsemisimple (if $w_{n} \neq 0$ ) but that semisimplicity is codimension one.

Example 3.5 Let $G=\operatorname{Sp}(2 n, \mathbb{R})$ be the group of $2 n \times 2 n$ symplectic matrices. The Lie algebra $L G$ consists of the infinitesimally symplectic matrices

$$
\left(\begin{array}{cc}
A & B \\
C & -A^{T}
\end{array}\right),
$$

where $B$ and $C$ are symmetric $n \times n$ matrices and $A$ is a general matrix. It is a well known and elementary that eigenvalues of $\xi \in L G$ occur in quadruplets $\pm \mu, \pm \bar{\mu}$, $\mu \in \mathbb{C}$. It follows that if we define $U_{T}$ to be the set of those $\xi \in L G$ for which the eigenvalues of $\xi$ are simple, purely imaginary and nonzero, then $U_{T}$ is nonempty and open in $L G$. Of course, if $U_{R}$ is the set of those elements $\xi$ whose eigenvalues all have nonzero real part, then $U_{R}$ is also nonempty and open. Moreover, $\xi \in U_{R}$ implies that $K(\xi) \cong \mathbb{R}$ and $\xi \in U_{T}$ implies that $K(\xi)$ is compact. It follows that neither the maximal torus or the line is preferred: $\operatorname{codim} \mathbf{T}^{d}=\operatorname{codim} \mathbb{R}=0$. 
A similar class of examples is provided by the groups $\mathbf{S O}(p, q), p \geq q \geq 0$ consisting of matrices that preserve the quadratic form $x_{1}^{2}+\cdots+x_{p}^{2}-x_{p+1}^{2}-\cdots-x_{p+q}^{2}$. We have the special cases $\mathbf{S O}(p, 0)=\mathbf{S O}(p)$ compact and $\mathbf{S O}(1,1) \cong \mathbb{R}$. In all other cases, $\operatorname{codim} \mathbf{T}^{d}=\operatorname{codim} \mathbb{R}=0$. The Lorentz group corresponds to the case $p=3$, $q=1$.

Example 3.6 Let $G=\mathrm{SL}(n, \mathbb{R}), n \geq 3$. This is homeomorphic as a topological space to $\mathbf{S O}(n) \times \mathbb{R}^{n(n+1) / 2-1}$ and $\operatorname{rank}(G)=\operatorname{rank}(\mathbf{S O}(n))=[n / 2]$.

The Lie algebra $L G$ consists of trace-zero matrices. Consider the open subset $U$ of elements $\xi \in L G$ for which all eigenvalues have nonzero real part. Since $n \geq 3$ and the only restriction in $L G$ is that elements have trace zero, it is easily seen that $U$ is also dense with full measure.

This argument fails when $n=2$. Indeed $\operatorname{SL}(2, \mathbb{R}) \cong \operatorname{Sp}(2, \mathbb{R})$ and we refer to Example 3.5.

We end by stating a result on direct products.

Proposition 3.7 Suppose that $G=G_{1} \times G_{2}$. Then the ranks satisfy $d=d_{1}+d_{2}$ and

(a) $\operatorname{codim}_{G} \mathbf{T}^{d}=\operatorname{codim}_{G_{1}} \mathbf{T}^{d_{1}}+\operatorname{codim}_{G_{2}} \mathbf{T}^{d_{2}}$.

(b) $\operatorname{codim}_{G} \mathbb{R}=\min \left(\operatorname{codim}_{G_{1}} \mathbb{R}, \operatorname{codim}_{G_{2}} \mathbb{R}\right)$.

Proof This follows from the observation that $K(\xi)$ is compact for $\xi=\xi_{1}+\xi_{2} \in$ $L G_{1} \oplus L G_{2}$ if and only if $K\left(\xi_{1}\right)$ and $K\left(\xi_{2}\right)$ are both compact.

\section{Cartan subgroups in noncompact Lie groups}

The results of Section 2 for connected Lie groups are sufficient for our intended applications on relative equilibria. To understand relative periodic orbits, we must consider the case when the Lie group $G$ is nonconnected as well as noncompact.

First suppose that $G$ is compact (but not necessarily connected). Let $H(g)$ denote the closure of the group generated by $g$. Such a subgroup $H(g)$ is called topologically cyclic (or 'monogenic') and is a compact abelian group. Hence $H(g) \cong \mathbf{T}^{p} \times \mathbb{Z}_{k}$. The subgroup $H(g)$ is a Cartan subgroup if $H(g)$ is maximal in the sense that there is no higher dimensional topologically cyclic subgroup of $G$ containing $H(g)$.

Note that it is possible for one Cartan subgroup to be properly contained in another. For example, consider the group $G=\mathbf{S O}(2) \times \mathbb{Z}_{2}$. The topologically cyclic subgroups are given by

$$
\mathbf{S O}(2) \times \mathbb{Z}_{2}, \quad \mathbf{S O}(2) \times \mathbf{1}, \quad \mathbb{Z}_{k} \times \mathbb{Z}_{2}(k \text { odd }), \quad \mathbb{Z}_{k} \times \mathbf{1} .
$$


The Cartan subgroups are $\mathbf{S O}(2) \times \mathbb{Z}_{2}$ and $\mathbf{S O}(2) \times \mathbf{1}$.

A second instructive example is the group $G=\mathbf{O}(2)$. The topologically cyclic subgroups are

$$
\mathbf{S O}(2), \quad \mathbb{Z}_{k}, \quad \mathbb{D}_{1},
$$

where $\mathbb{D}_{1}$ is the two element group generated by a reflection in $\mathbf{O}(2)-\mathbf{S O}(2)$. Although there are infinitely many copies of $\mathbb{D}_{1}$ in $\mathbf{O}(2)$, they are all conjugate. Again. there are two Cartan subgroups up to conjugacy: $\mathbf{S O}(2)$ and $\mathbb{D}_{1}$.

For general $G$ compact, we have the projection $p: G \rightarrow G / G^{0}$ where $G^{0}$ is the connected component of the identity in $G$. In the above examples, $G / G^{0} \cong \mathbb{Z}_{2}$. For each of the two cyclic subgroups $C \subset G / G^{0}$, there exists a unique (up to conjugacy) Cartan subgroup $H \subset G$ such that $p(H)=C$. These examples generalize as follows, see for example [5, Chapter IV, Proposition 4.6].

Theorem 4.1 Suppose that $G$ is a compact Lie group with connected component of the identity $G^{0}$. There is a one-to-one correspondence between conjugacy classes of cyclic subgroups $C$ of $G / G^{0}$ and conjugacy classes of Cartan subgroups $H$ in $G$ satisfying $p(H)=C$.

More precisely, if $C$ is a cyclic subgroup of $G / G^{0}$, then there is a Cartan subgroup $H \subset G$ with $p(H)=C$. The subgroup $H$ is isomorphic to $\mathbf{T}^{p} \times C$. If $H^{\prime}$ is a Cartan subgroup with $p\left(H^{\prime}\right)$ conjugate to $C$, then $H^{\prime}$ is conjugate to $H$.

This theorem generalizes the standard results on maximal tori (the Cartan subgroups corresponding to $C=\mathbf{1}$ are the maximal tori in $\left.G^{0}\right)$.

As in [10], given a cyclic subgroup $C \subset G / G^{0}$, we define $\operatorname{rank}(G, C)=d$ where $d$ is the dimension of the Cartan subgroup corresponding to $C$. Thus $\operatorname{rank}(G, \mathbf{1})=$ $\operatorname{rank}(G)$. In our examples,

$$
\operatorname{rank}\left(\mathbf{S O}(2) \times \mathbb{Z}_{2}, \mathbb{Z}_{2}\right)=1, \quad \operatorname{rank}\left(\mathbf{O}(2), \mathbb{Z}_{2}\right)=0
$$

Now we drop the assumption that $G$ is compact. We require that $G$ has finitely many connected components. Again, there is a maximal compact subgroup $K$ that is unique up to conjugacy and such that $G$ is diffeomorphic to $K \times \mathbb{R}^{n}$ for some $n$. It is easily seen (arguing as in Proposition 2.1) that every topologically cyclic subgroup $H \subset G$ is either compact (hence conjugate to a subgroup of a Cartan subgroup of $K$ ) or isomorphic to $\mathbb{Z}$. We say that $H$ is a Cartan subgroup of $G$ if $H$ is conjugate to a Cartan subgroup of $K$. We have the following analogue to Corollary 2.2.

Corollary 4.2 Let $G$ be a finite dimensional Lie group with finitely many connected components. Either $H(g)$ is contained in a Cartan subgroup or $H(g) \cong \mathbb{Z}$. 
Proposition 4.3 The quotients $G / G^{0}$ and $K / K^{0}$ are isomorphic. In particular, there is a one-to-one correspondence between conjugacy classes of cyclic subgroups $C$ of $G / G^{0}$ and conjugacy classes of Cartan subgroups of $G$. Moreover, if $H \subset G$ is a Cartan subgroup corresponding to the cyclic subgroup $C \subset G / G^{0}$, then $p(H)=C$ and $H \cong \mathbf{T}^{p} \times C$.

Proof We have the group isomorphism

$$
K / K^{0}=K /\left(G^{0} \cap K\right) \cong\left(G^{0} K\right) / G^{0}=G / G^{0} .
$$

Theorem 4.4 Suppose that $G$ is a finite dimensional Lie group with finitely many connected components. For almost every $g \in G$, the subgroup $H(g)$ is conjugate either to a Cartan subgroup or to a copy of $\mathbb{Z}$.

Proof Fix $\alpha \in G / G^{0}$ and let $C$ be the cyclic subgroup of $G / G^{0}$ generated by $\alpha$. Let $\mathrm{T}^{d} \times C$ denote the corresponding Cartan subgroup of $G$. Since $G / G^{0}$ is finite, we can restrict attention to elements $g \in G(\alpha)$ where $G(\alpha)=\{g \in G, p(g)$ is conjugate to $\alpha\}$. We show that $H(g) \cong \mathbf{T}^{d} \times C$ or $\mathbb{Z}$ for almost every $g \in G(\alpha)$.

Define

$$
S=\{g \in G(\alpha), H(g) \text { is compact but not Cartan }\} .
$$

Let $S_{0}=\left(\mathbf{T}^{d} \times C\right) \cap S$ and consider the conjugation map $\rho: G /\left(\mathbf{T}^{d} \times C\right) \times\left(\mathbf{T}^{d} \times C\right) \rightarrow$ $G$. The remainder of the proof is identical to the proof of Lemma 2.5.

Let $T^{d} \times C$ denote the Cartan subgroup of $G$ corresponding to the cyclic subgroup $C \subset G / G^{0}$. We define $\operatorname{rank}(G, C)=d$. Note that $\operatorname{rank}(G, C)=\operatorname{rank}\left(K, C^{\prime}\right)$ where $C^{\prime}$ is the corresponding cyclic subgroup of $K / K^{0}$.

Example 4.5 Let $G=\mathbf{E}(2)$. Then $G / G^{0} \cong \mathbb{Z}_{2}$ and we have the cyclic subgroups $C=\mathbf{1}$ and $C=\mathbb{Z}_{2}$. The maximal compact subgroup of $G$ is given by $K=\mathbf{O}(2)$. Since $\operatorname{rank}(\mathbf{O}(2), C)=1$ when $C=\mathbf{1}$ and 0 when $C=\mathbb{Z}_{2}$, we have

$$
\operatorname{rank}(\mathbf{E}(2), \mathbf{1})=1 \quad \operatorname{rank}\left(\mathbf{E}(2), \mathbb{Z}_{2}\right)=0 .
$$

Now suppose that $g \in \mathbf{E}(2)$ with corresponding topologically cyclic subgroup $H(g)$. Let $C \subset \mathbb{Z}_{2}$ be the cyclic subgroup generated by $p(g)$. If $C=\mathbf{1}$ then it follows from Theorem 4.4 that generically either $H(g) \cong \mathrm{T}^{1}$ or $H(g) \cong \mathbb{Z}$. If $C=\mathbb{Z}_{2}$, then generically either $H(g) \cong \mathbb{Z}_{2}$ or $H(g) \cong \mathbb{Z}$. There is the question of the codimensions of these events. 
Generally, consider those $g \in G$ such that $p(g)$ generates the cyclic subgroup $C$. Let $\operatorname{codim}_{(G, C)} \mathbf{T}^{d}$ denote the codimension of the event that $H(g) \cong \mathbf{T}^{d} \times C$ where $d=\operatorname{rank}(G, C)$ and let $\operatorname{codim}_{(G, C)} \mathbb{Z}$ denote the codimension of the event that $H(g) \cong \mathbb{Z}$. When $C=\mathbf{1}$, it is easily seen that

$$
\operatorname{codim}_{(G, \mathbf{1})} \mathbf{T}^{d}=\operatorname{codim}_{G} \mathbf{T}^{d}, \quad \operatorname{codim}_{(G, \mathbf{1})} \mathbb{Z}=\operatorname{codim}_{G} \mathbb{R} .
$$

For $G=\mathbf{E}(2)$, we have

$\operatorname{codim}_{(\mathbf{E}(2), \mathbf{1})} \mathbf{T}^{1}=0, \quad \operatorname{codim}_{(\mathbf{E}(2), \mathbf{1})} \mathbb{Z}=1, \quad \operatorname{codim}_{\left(\mathbf{E}(2), \mathbb{Z}_{2}\right)} \mathbf{T}^{1}=1, \quad \operatorname{codim}_{\left(\mathbf{E}(2), \mathbb{Z}_{2}\right)} \mathbb{Z}=0$.

To verify the last two codimensions, let $g \in \mathbf{E}(2)$ be an element such that $p(g)$ is nontrivial. In other words, $g$ is a reflection composed with a translation. Fix a reflection $\kappa \in \mathbf{O}(2)-\mathbf{S O}(2)$. It is an elementary fact that $g$ is conjugate to $\kappa$ composed with a translation $v$ parallel to the axis of symmetry of $\kappa$. (If $v=0, g$ is a reflection; if $v \neq 0, g$ is a glide-reflection.) Observe that $H(g) \cong \mathbb{Z}$ if and only if $v \neq 0$ (codimension zero). If $v=0$ (codimension one) then $H(g) \cong \mathbb{Z}_{2}$.

Example 4.6 Let $G=\mathbf{E}(3)$. Again $G / G_{0} \cong \mathbb{Z}_{2}$. Using results for the maximal compact subgroup $K=\mathbf{O}(3)$, we compute that

$$
\operatorname{rank}(\mathbf{E}(3), \mathbf{1})=1 \quad \operatorname{rank}\left(\mathbf{E}(3), \mathbb{Z}_{2}\right)=1 .
$$

It follows from the results for $G=\mathbf{S E}(3)$ in Example 3.4 that

$$
\operatorname{codim}_{(\mathbf{E}(3), \mathbf{1})} \mathbf{T}^{1}=1, \quad \operatorname{codim}_{(\mathbf{E}(3), \mathbf{1})} \mathbb{Z}=0 .
$$

We claim that

$$
\operatorname{codim}_{\left(\mathbf{E}(3), \mathbb{Z}_{2}\right)} \mathbf{T}^{1}=0, \quad \operatorname{codim}_{\left(\mathbf{E}(3), \mathbb{Z}_{2}\right)} \mathbb{Z}=1 .
$$

Suppose that $g=(A, v) \in \mathbf{E}(3)$ with $p(g)$ nontrivial. The orthogonal matrix $A$ has one eigenvalue equal to -1 and a pair of complex eigenvalues. Generically, these eigenvalues are not equal to 1 , in which case $g$ is conjugate to the element $(A, 0) \in$ $\mathbf{E}(3)$. It follows that $\operatorname{codim}_{\left(\mathbf{E}(3), \mathbb{Z}_{2}\right)} \mathbf{T}^{1}=0$. In a one-parameter family $A$ may have eigenvalues equal to 1 in which case $g$ is a reflection. After conjugacy, $v$ is any translation parallel to the reflection plane. Generically $v \neq 0$ and $H(g) \cong \mathbb{Z}$ so that $\operatorname{codim}_{\left(\mathbf{E}(3), \mathbb{Z}_{2}\right)} \mathbb{Z}=1$.

To summarize, when $C=\mathbf{1}$, generically $H(g) \cong \mathbf{T}^{1}$ and $H(g) \cong \mathbb{Z}$ occurs with codimension one. When $C=\mathbb{Z}_{2}$, generically $H(g) \cong \mathbb{Z}$ and $H(g) \cong \mathbf{T}^{1} \times \mathbb{Z}_{2}$ occurs with codimension one. 


\section{Dynamical systems with noncompact symmetry}

Noncompact symmetry groups arise naturally in dynamical systems, especially for infinite dimensional evolution equations on unbounded domains. In the remainder of this paper, we describe the structure of relative equilibria and relative periodic orbits when there is a noncompact symmetry group. In particular, under certain technical hypotheses, we show that the drift phenomena found by Field [9] and Krupa [14] have counterparts for evolution equations on unbounded domains. An important extra hypothesis we require is that the patterns concerned do not possess what we call approximate symmetries, see below.

Throughout the remainder of this paper, smooth means $C^{k}$ for some $k \geq 1$. We do not require that the symmetry group acts smoothly on the full infinite-dimensional space.

First, we recall some basic definitions and results regarding infinite dimensional dynamical systems. For details we refer to Henry [12]. Suppose that $\mathcal{B}$ is a Banach space. Let $A: \mathcal{B} \rightarrow \mathcal{B}$ be a linear (unbounded) operator that generates an analytic semigroup (semiflow) on $\mathcal{B}$. A necessary and sufficient condition for this is that $A$ is 'sectorial'. In particular, the domain $D(A)$ of $A$ is dense in $\mathcal{B}$. Let $N$ be any (nonlinear, unbounded) operator on $\mathcal{B}$. We suppose that $N$ is smooth $\left(C^{k}\right.$ for some $k \geq 1$ ) when viewed as an operator $N: D\left(A^{\alpha}\right) \rightarrow \mathcal{B}$ for some $\alpha \in[0,1)$ and that $(d N)_{0}=0$. Then $f=A+N$ is a smooth 'vector field' on $\mathcal{B}$ and generates a local semidynamical system (enjoying the usual properties such as existence and uniqueness, smooth dependence on initial conditions, smoothness of solution trajectories, and so on).

Now suppose that $\Gamma$ is a (noncompact) finite dimensional Lie group acting linearly, but not necessarily smoothly, on $\mathcal{B}$. As usual, we say that the vector field $f$ is $\Gamma$ equivariant if $f(\gamma x)=\gamma f(x)$ for all $\gamma \in \Gamma$ and $x \in D(A)$. Then $f$ induces a smooth $\Gamma$-equivariant semiflow consisting of solutions to the initial value problem

$$
\dot{x}=f(x), \quad x(0)=x_{0} .
$$

The action of $\Gamma$ on $\mathcal{B}$ induces an action of the Lie algebra $L \Gamma$ on $\mathcal{B}$ in the usual way:

$$
\xi x=\left.\frac{d}{d t} \exp (t \xi) x\right|_{t=0} .
$$

In general, the linear operator $\xi: \mathcal{B} \rightarrow \mathcal{B}$ is unbounded.

Definition 5.1 Let $x_{0} \in D(A)$ and set $X=\Gamma x_{0}$. The group orbit $X$ is called a relative equilibrium if the semiflow induced by $f$ leaves $X$ invariant. Equivalently, $f\left(x_{0}\right)=\xi x_{0}$ for some $\xi \in L \Gamma$. 
We define the isotropy subgroup of $X$ to be the isotropy subgroup of the point $x_{0}$. Since the isotropy subgroup of $\gamma x_{0}$ is $\gamma \Sigma \gamma^{-1}$, the isotropy subgroup of a relative equilibrium is well-defined up to conjugacy.

When $\Gamma$ is compact and $\mathcal{B}$ is finite dimensional, the relative equilibrium $X=\Gamma x_{0}$ is a manifold diffeomorphic to the homogeneous space $\Gamma / \Sigma$. This is not automatically the case in our setting and we require some extra hypotheses.

Definition 5.2 Suppose that there is a sequence $\left\{\gamma_{n}\right\} \in \Gamma / \Sigma$ which diverges to infinity (that is, there are no convergent subsequences) and possesses the property that $\gamma_{n} x_{0} \rightarrow x_{0}$. Then we say that $\left\{\gamma_{n}\right\}$ is an approximate symmetry of $x_{0}$.

Let $\mathcal{B}^{\prime}$ denote the subset of $D(A)$ consisting of those points $x \in D(A)$ with no approximate symmetries and such that the map $\gamma \mapsto \gamma x$ is smooth. Observe that $\mathcal{B}^{\prime}$ is a $\Gamma$-invariant subset of $\mathcal{B}$. When $\Gamma$ acts isometrically on $\mathcal{B}$, it is easy to see that having no approximate symmetries is an open condition.

Proposition 5.3 Suppose that $x_{0} \in \mathcal{B}^{\prime}$ with isotropy subgroup $\Sigma$. Then $X=\Gamma x_{0}$ is a submanifold of $\mathcal{B}$ diffeomorphic to $\Gamma / \Sigma$.

Proof Since the action of $\Gamma$ on $x_{0}$ is smooth, the group orbit $X$ is a smoothly immersed submanifold of $\mathcal{B}$, the tangent space at $x \in X$ being given by $T_{x} X=(L \Gamma) x$. The condition that there are no approximate symmetries guarantees that $X$ is an embedded submanifold.

\section{Relative equilibria}

In this section, we present our generalization of the results of Field [9] concerning the dynamics on relative equilibria. Upper bounds for the drift are given in Subsection 6.1. In Subsection 6.2, we prove that under certain reasonable hypotheses, these upper bounds (taken collectively) are attained. Moreover, the preferred upper bound (if it exists) is dictated by the genericity results at the Lie algebra level, as discussed in Section 3.

\subsection{An upper bound for the drift on a relative equilibrium}

We assume the setting described in Section 5. Thus, $\Gamma$ is a finite dimensional Lie group acting on the Banach space $\mathcal{B}$ and $f$ is an nonlinear (unbounded) operator defining a smooth $\Gamma$-equivariant local semiflow on $\mathcal{B}$. Let $x_{0}$ be a point with trivial isotropy lying in the subset $\mathcal{B}^{\prime}$ defined after Definition 5.2 and suppose that $X=\Gamma x_{0}$ is a relative equilibrium. 
Recall that $\operatorname{Fix}(\Sigma)$ is a flow-invariant subspace and that the largest subgroup of $\Gamma$ that acts on $\operatorname{Fix}(\Sigma)$ is the normalizer $N(\Sigma)$.

Proposition 6.1 Suppose that $x_{0} \in \mathcal{B}^{\prime}$ has isotropy subgroup $\Sigma$ and that $X=\Gamma x_{0}$ is a relative equilibrium. Then $f\left(x_{0}\right)=\xi x_{0}$ for some $\xi \in L(N(\Sigma))$ and the solution to the initial value problem (5.1) with initial condition $x_{0}$ is given by $x(t)=\exp (t \xi) x_{0}$.

Proof This is standard, see $[9,14]$. We reproduce the proof for completeness. Since $X$ is flow-invariant, $f\left(x_{0}\right)$ is tangent to $X$ and lies in $T_{x_{0}} X=L \Gamma x_{0}$. Hence $f\left(x_{0}\right)=\xi x_{0}$ for some $\xi \in L \Gamma$. Now set $x(t)=\exp (t \xi) x_{0}$. We compute that

$$
\begin{aligned}
\frac{d}{d t} x(t) & =\left.\frac{d}{d s} \exp (s \xi) x_{0}\right|_{s=t} \\
& =\left.\exp (t \xi) \frac{d}{d s} \exp ((s-t) \xi) x_{0}\right|_{s=t} \\
& =\left.\exp (t \xi) \frac{d}{d s} \exp (s \xi) x_{0}\right|_{s=0} \\
& =\exp (t \xi) \xi x_{0}=\exp (t \xi) f\left(x_{0}\right) \\
& =f\left(\exp (t \xi) x_{0}\right)=f(x(t))
\end{aligned}
$$

It remains to show that $\xi \in L(N(\Sigma))$. The isotropy subgroup of $x(t)=\exp (t \xi) x_{0}$ is given by $\Sigma_{t}=\exp (t \xi) \Sigma \exp (t \xi)^{-1}$ so that $x(t) \in \operatorname{Fix}\left(\Sigma_{t}\right)$ for each $t$. But flowinvariance of $\operatorname{Fix}(\Sigma)$ implies that $x(t)$ has isotropy subgroup $\Sigma$ for all $t$. Hence $\Sigma_{t} \equiv \Sigma$ from which we deduce that $\exp (t \xi) \in N(\Sigma)$ for all $t$. Since $\exp : L \Gamma \rightarrow \Gamma$ is a diffeomorphism on some neighborhood of the origin, it follows that $t \xi \in L(N(\Sigma))$ for $t$ small enough and hence that $\xi \in L(N(\Sigma))$.

Let $X_{0}$ denote the closure of the trajectory through $x_{0}$. Then $X_{0}=K(\xi) x_{0}$ for some $\xi \in L(N(\Sigma))$ and is minimal: there are no proper closed flow-invariant subsets in $X_{0}$. Moreover $X_{0} \subset \operatorname{Fix}(\Sigma)$. Since the relative equilibrium $X$ is foliated by the minimal subsets $\gamma X_{0}, \gamma \in \Gamma$, it follows that the flow on $X$ is completely determined by the flow on $X_{0} \subset \operatorname{Fix}(\Sigma)$.

The group $N(\Sigma)$ acts on $\operatorname{Fix}(\Sigma)$, the kernel of the action being given by $\Sigma$. Hence, we can write $X_{0}=K(\xi) x_{0}$ with $\xi \in L(N(\Sigma) / \Sigma)$. In particular, $K(\xi)$ is the closure of a one parameter subgroup in $N(\Sigma) / \Sigma$. The following result is now immediate by Corollary 2.2.

Corollary 6.2 Suppose that the hypotheses of Proposition 6.1 are valid. Then the relative equilibrium $X$ is foliated by minimal closed flow-invariant subsets conjugate to $X_{0}=K(\xi) x_{0}$ and consisting either of tori $\mathbf{T}^{p}, p \leq \operatorname{rank}(N(\Sigma) / \Sigma)$, or of copies of $\mathbb{R}$. 


\subsection{Generic attainment of the upper bounds}

In this subsection, we prove that the upper bounds established in Subsection 6.1 are generically attained provided we impose extra technical hypotheses on the structure of $\mathcal{B}$. We continue to assume that $f: \mathcal{B} \rightarrow \mathcal{B}$ is a $\Gamma$-equivariant vector field with relative equilibrium $X=\Gamma x_{0}$ where $x_{0} \in \mathcal{B}^{\prime}$. As usual, $\Sigma$ is the isotropy subgroup of $x_{0}$. Let $\mathcal{Z}$ denote the space of smooth $\left(C^{k}\right) \Gamma$-equivariant vector fields on $\mathcal{B}$ and let $\mathcal{Z}_{X}$ denote the subspace of vector fields for which $X$ is a relative equilibrium. For any $k \geq 1, \mathcal{Z}$ is a complete metric space in the $C^{k}$ topology and $\mathcal{X}_{Z}$ is a closed subspace. (We do not assume that $f \in \mathcal{Z}-f$ may be an unbounded operator.)

Theorem 6.3 Suppose that $\mathcal{B}$ is a Hilbert space and that the action of $\Gamma$ on $\mathcal{B}$ is unitary. Then there is a residual subset $\mathcal{R} \subset \mathcal{Z}_{X}$ such that for all $h \in \mathcal{R}$, the minimal $(f+h)$-flow-invariant subsets in $X$ are maximal tori in $N(\Sigma) / \Sigma$ (with codimension $\operatorname{codim}_{N(\Sigma) / \Sigma} \mathbf{T}^{d}$ ) or copies of $\mathbb{R}$ (with codimension $\operatorname{codim}_{N(\Sigma) / \Sigma} \mathbb{R}$ ).

The main step in the proof of Theorem 6.3 is the following.

Lemma 6.4 Suppose that $\mathcal{B}$ is a Hilbert space and that the action of $\Gamma$ is unitary. Let $x_{0}$ be a point in $\mathcal{B}^{\prime}$ with isotropy $\Sigma$ and let $U$ be a neighborhood of $\Gamma x_{0}$. Set $V=\left\{h\left(x_{0}\right): h \in \mathcal{Z}_{X}, \operatorname{supp} h \subset U\right\}$. Then $L(N(\Sigma) / \Sigma) x_{0}=V$.

Proof The inclusion $V \subset L(N(\Sigma) / \Sigma) x_{0}$ is clear from Proposition 6.1. To prove the reverse inclusion, we follow an argument in Krupa [14].

By Proposition 5.3, $X=\Gamma x_{0}$ is a submanifold of $\mathcal{B}$ and we can construct the normal bundle $N(X)$. Since the action of $\Gamma$ is unitary, $N(X)$ is $\Gamma$-invariant. The usual arguments show that $N(X)$ is locally diffeomorphic to $\mathcal{B}$ and the homogeneity of the group orbit $X$ ensures that there is neighborhood of $X$ in $N(X)$ that is equivariantly diffeomorphic to a $\Gamma$-invariant neighborhood $U^{\prime}$ of $X$ in $\mathcal{B}$. Identify $T N(X)$ with $\mathcal{B}$. Without loss of generality, we may suppose that $U=U^{\prime}$.

Let $\xi \in L(N(\Sigma) / \Sigma)$. We construct a map $h \in \mathcal{Z}$ supported in $U$ with $h\left(x_{0}\right)=\xi x_{0}$. Choose $\epsilon>0$ so that the ball in $N_{x_{0}}$ of radius $\epsilon$ and center $x_{0}$ is contained in $U \cap N_{x_{0}}$. Let $b:[0, \infty] \rightarrow[0,1]$ be a smooth bump function with $\left.b\right|_{[0, \epsilon / 3]} \equiv 1$ and $\left.b\right|_{[2 \epsilon / 3, \infty]} \equiv 0$. We define $h(x)=b\left(\left\|x-x_{0}\right\|\right) \xi x_{0}$. The map $h: N_{x_{0}} \rightarrow \mathcal{B}$ is supported inside $N_{x_{0}} \cap U$, is constant in a neighborhood of $x_{0}$ with the required value $\xi x_{0}$ and is smooth away from $x_{0}$ by smoothness of the norm. Moreover, $h$ is $\Sigma$-equivariant since the norm is invariant and $x_{0}, \xi x_{0} \in \operatorname{Fix}(\Sigma)$. Indeed, $h(\sigma x)=h(x)=\sigma h(x)$ for all $x \in N_{x_{0}}, \sigma \in \Sigma$.

There is a unique $\Gamma$-equivariant extension of $h$ from $N_{x_{0}}$ to the whole of $N(X)$ given explicitly by $h(x)=b\left(\left\|x-\gamma x_{0}\right\|\right) \operatorname{Ad}_{\gamma} \xi \gamma x_{0}$ for all $x \in \gamma N_{x_{0}}$. The fact that this extension is well-defined is due to the $\Sigma$-equivariance of $h$ on $N_{x_{0}}$. Moreover, $h$ is 
smooth since $\Gamma$ acts smoothly on $x_{0}$. This completes the construction of the required function $h$.

The proof of Lemma 6.4 shows that $h \in \mathcal{Z}_{X}$ can be chosen so that $\|h\|_{C^{k}} \leq$ $M\left\|h\left(x_{0}\right)\right\|$ where $M$ is a constant depending only on the choice of bump function $b$. Theorem 6.3 follows immediately from Theorem 2.4, Corollary 6.2 and Lemma 6.4. Since 'almost all' in Theorem 2.4 holds both in the topological and measure-theoretic senses, it follows similarly that the set $\mathcal{R}$ in Theorem 6.3 is prevalent in the sense of Hunt et al [13].

Theorem 6.3 can be refined somewhat to circumvent the distinguished roles played by $f$ and $X$. To avoid technical difficulties associated with the infinite dimensional setting, we suppose that the group and the evolution operators act smoothly on the whole of $\mathcal{B}$. In addition, we suppose that $\mathcal{B}$ is a Hilbert space and that the action of $\Gamma$ is unitary. Finally, we let $\widetilde{\mathcal{Z}}$ consist of those smooth vector fields for which all relative equilibria are normally hyperbolic. It follows from the generalization in $[11$, Appendix] of results of Krupa [14] that $\widetilde{\mathcal{Z}}$ is an open subset of $\mathcal{Z}$ and that the relative equilibria of $f \in \widetilde{\mathcal{Z}}$ are isolated and persist under perturbation. (Since $\mathcal{B}$ is infinite dimensional, $\widetilde{\mathcal{Z}}$ is not a dense subset of $\mathcal{Z}$.)

Theorem 6.5 Under the above simplifying assumptions, there is a residual (and prevalent) subset $\mathcal{R} \subset \widetilde{\mathcal{Z}}$ such that for every $f \in \mathcal{R}$, each relative equilibrium is foliated by minimal flow-invariant subsets consisting of either maximal tori or lines.

\section{Relative periodic orbits}

We now show, under similar hypotheses to those made in the previous section, that the results of Krupa [14] and Field [10] for relative periodic orbits carry over to evolution equations with noncompact symmetry group. Suppose that $P \subset \mathcal{B}^{\prime}$ is a relative periodic orbit. That is, $P$ is $\Gamma$-invariant and flow-invariant, the orbit space $P / \Gamma$ is diffeomorphic to $S^{1}$ and the induced flow on the orbit space is nontrivial (hence periodic). Let $\pi: P \rightarrow S^{1}$ denote the projection.

Choose a point $x_{0} \in P$ and let $x(t)$ be the solution curve with $x(0)=x_{0}$. Then $\pi(x(t+T))=\pi(x)$ where $T$ is the period of the periodic orbit in the orbit space. Hence $x(t+T)=\gamma x(t)$ for some $\gamma \in \Gamma$. In fact, if $x_{0}$ has isotropy $\Sigma$, then $\gamma \in N(\Sigma)$ and $\gamma$ is identified with a unique element of $N(\Sigma) / \Sigma$. Let $H=H(\gamma)$ denote the corresponding topologically cyclic subgroup of $N(\Sigma) / \Sigma$. Since $x_{0} \in \mathcal{B}^{\prime}$, the group orbit $H x_{0}$ is a submanifold of the fiber $\pi^{-1}\left(\pi\left(x_{0}\right)\right)$ and $H x_{0}$ is diffeomorphic to $H$.

Proposition 7.1 The relative periodic orbit is foliated by tori of dimension $\operatorname{dim} H+1$ (with irrational tori flow) or by copies of $\mathbb{R}$ (with unbounded linear flow). 
Proof (cf Field [9, p. 189]) Let $P_{0}$ denote the closure of the trajectory $x(t)$ inside $P$. The submanifold $H x_{0}$ consists of those $x \in P_{0}$ such that $\pi(x)=\pi\left(x_{0}\right)$. In particular, $H x_{0}$ is a cross-section for the flow in $P_{0}$ with Poincaré map $h x_{0} \mapsto \gamma h x_{0}$. Conversely, the flow in $P_{0}$ is the suspension of the Poincaré map. In particular, we have

$$
P_{0}=H x_{0} \times \mathbb{R} / \sim
$$

where $\sim$ is defined by $\left(h x_{0}, t+T\right) \sim\left(\gamma h x_{0}, t\right)$.

It follows that $P_{0}$ is diffeomorphic to the suspension $H \times \mathbb{R} / \sim$ and it is easily verified that the group structure on $H \times \mathbb{R}$ induces a group structure on $H \times \mathbb{R} / \sim$. Moreover, the trajectory $x(t)$ in $P_{0}$ is identified with a dense one-parameter subgroup in $H \times \mathbb{R} / \sim$. It follows (as in Proposition 2.1) that $H \times \mathbb{R} / \sim$ is either a torus $\mathbf{T}^{p}$ or a line $\mathbb{R}$. Moreover the flow on $H \times \mathbb{R} / \sim$ and hence on $P_{0}$ is as required. Finally, $\operatorname{dim} P_{0}=\operatorname{dim} H+1$.

The results of Section 4 give an upper bound for the dimension in Proposition 7.1. Let $G=N(\Sigma) / \Sigma$ and let $p: G \rightarrow G / G^{0}$ be the projection where $G^{0}$ is the connected component of the identity in $G$. Suppose that $\gamma \in G$ is as defined above and let $C$ be the cyclic subgroup of $G / G^{0}$ generated by $p(\gamma)$. Recall that $\operatorname{rank}(G, C)$ is the dimension of the corresponding Cartan subgroup of $G$. We now have the following generalization of a result of Krupa [14] (see also Field [10]).

Corollary 7.2 Suppose that $P$ is a relative periodic orbit containing a point $x_{0} \in \mathcal{B}^{\prime}$ with isotropy $\Sigma$. Let $C$ be the associated cyclic group as defined above. Then $P$ is foliated by tori $\mathbf{T}^{p}, p \leq \operatorname{rank}(N(\Sigma) / \Sigma, C)+1$, or by copies of $\mathbb{R}$.

In applications, it is convenient to characterize the cyclic subgroup $C$ as follows. Suppose that $P$ is a relative periodic orbit containing a point $x_{0}$ with isotropy $\Sigma$ and let $P_{0}$ be the closure of the trajectory through $x_{0}$. Define $\widetilde{\Sigma}$ to be the symmetry group of the set $P_{0}$

$$
\widetilde{\Sigma}=\left\{\gamma \in \Gamma: \gamma P_{0}=P_{0}\right\}
$$

Observe that $\Sigma$ is a normal subgroup of $\widetilde{\Sigma}$ and $\widetilde{\Sigma} / \Sigma$ is a topologically cyclic subgroup of $G=N(\Sigma) / \Sigma$. Let $p: G \rightarrow G^{0}$ be the projection. Then $C=p(\widetilde{\Sigma})$.

The subgroup $\widetilde{\Sigma}$ has the following physical interpretation. Since we are working with a relative periodic orbit rather than a relative equilibrium, the initial state $x_{0}$ evolves into states that are not symmetrically related to $x_{0}$. However it may be the case that after a certain period of time, the solution reaches a state $x\left(T^{\star}\right)$ such that $x\left(T^{\star}\right)=\sigma^{\star} x_{0}$ where $\sigma^{\star} \neq 1$. Such a solution is often called a discrete rotating wave and $\widetilde{\Sigma}$ is the subgroup of $\Gamma$ generated by such elements $\sigma^{\star}$.

More generally, the solution may vary quasiperiodically in time but may limit on a state $x^{\star}=\sigma^{\star} x_{0}$. Again, $\widetilde{\Sigma}$ is the group generated by these elements $\sigma^{\star}$. 
Theorem 7.3 Suppose that the hypotheses of Corollary 7.2 are valid. Suppose in addition that $\mathcal{B}$ is a separable Hilbert space and that the action of $\Gamma$ on $\mathcal{B}$ is unitary. Then generically, the relative periodic orbit $P$ is foliated by tori $\mathbf{T}^{p}$ of maximal dimension $p=\operatorname{rank}(N(\Sigma) / \Sigma, C)+1$, or by copies of $\mathbb{R}$.

Proof Again, we follow Field [9]. Construct the normal bundle $N(P)$ which is identified with $\mathcal{B}$. Let $D$ denote the restriction of $N(P)$ to the group orbit $\Gamma x_{0}$. Let $D^{\prime}$ denote an open subbundle of $D$ containing $\Gamma x_{0}$ and let $D^{\prime \prime}=D^{\prime} \cap D(A)$ (recall that $D(A)$ is the domain of the linear part of the vector field). The sets $D, D^{\prime}, D^{\prime \prime}$ are all $\Gamma$-invariant.

Let $\phi=\phi(x, t)$ denote the semiflow defined locally on $\mathcal{B}=N(P)$ by the underlying vector field $f$. Since the flow on the orbit space $S^{1}$ is assumed to be nontrivial, the semiflow on $N(P)$ is transverse to $D$ in a neighborhood of $P$. It follows from Henry [12] that we can choose $D^{\prime}$ small enough and a unique $\tau(x)$ close to $T$ such that $\phi(x, \tau(x)) \in D$ for each $x \in D^{\prime \prime}$. As usual, the map $\tau: D^{\prime \prime} \rightarrow \mathbb{R}$ is smooth and determines a smooth $\Gamma$-equivariant Poincaré map $\psi: D^{\prime \prime} \rightarrow D, \psi(x)=\phi(x, \tau(x))$.

As noted above, $\psi\left(x_{0}\right)=\gamma x_{0}$ for some $\gamma \in N(\Sigma)$ which is identified with a unique element $\gamma \in N(\Sigma) / \Sigma$. We claim that for a typical underlying vector field $f: \mathcal{B} \rightarrow \mathcal{B}$, the corresponding element $\gamma \in N(\Sigma) / \Sigma$ is typical. The theorem then follows from Theorem 4.4 and Proposition 7.1.

The claim follows from standard perturbation arguments $[9$, p. 198]. Let $\delta=$ $\exp \xi \in N(\Sigma)$ close to the identity. Let $D_{x_{0}}\left(D_{x_{0}}^{\prime \prime}\right)$ denote the fiber of the bundle $D\left(D^{\prime \prime}\right)$ over $x_{0}$. We have the isotopy $\chi: D_{x_{0}}^{\prime \prime} \times[0, T] \rightarrow D_{x_{0}}$ from inclusion to multiplication by $\delta$ given by $\chi(x, t)=b\left(\left\|x-x_{0}\right\|\right)(\exp t \xi / T) x$ where $b$ is a bump function defined as in the proof of Lemma 6.4. Once again, by smoothness of the action of $\Gamma$ on $x_{0}$, the map $\chi$ extends to an equivariant isotopy $\chi$ from $D^{\prime \prime}$ into $D$.

Now we define a perturbed equivariant 'flow'

$$
\tilde{\phi}(x, t)=\phi(\chi(x, t), t) \quad x \in D^{\prime \prime} \quad t \in[0, \rho(x)] .
$$

Let $\tilde{\psi}: D^{\prime \prime} \rightarrow D$ be the corresponding Poincaré map. We compute that

$$
\tilde{\phi}\left(x_{0}, T\right)=\phi\left(\chi\left(x_{0}, T\right), T\right)=\phi\left(\delta x_{0}, T\right)=\delta \phi\left(x_{0}, T\right) .
$$

Hence, $\tilde{\psi}\left(x_{0}\right)=\delta \psi\left(x_{0}\right)$ as required.

This essentially proves the claim. As defined, $\tilde{\phi}$ is continuous. Modifying the definitions of $\chi$ and $\tilde{\phi}$ near $t=0$ and $t=T$ we ensure that $\tilde{\phi}$ is $C^{k}$. Moreover, $\tilde{\phi}$ is $C^{k}$-close to $\phi$ for $\delta$ close to the identity. Of course, there is no reason why $\tilde{\phi}$ should satisfy the group law $\tilde{\phi}(x, t+s)=\tilde{\phi}(\tilde{\phi}(x, t), s)$. Nevertheless, we can define an equivariant vector field $\tilde{f}$ whose solution curves coincide as sets with the curves $\tilde{\phi}(x, \cdot)$. There is a loss of differentiability in defining $\tilde{f}$ this way: observe that $\phi$ is 
$C^{k}$ in $(x, t)$ but $C^{k+1}$ in $t$. However, $\tilde{\phi}$ is only $C^{k}$ even in $t$ and hence $\tilde{f}$ is $C^{k-1}$. This problem is easily circumvented by first $C^{k}$-approximating $f$ by a $C^{k+1}$ (say) vector field $f^{\star}$ and then $C^{k}$-approximating $f^{\star}$ by the $C^{k}$ vector field $\tilde{f}$. Finally, the separability of the Hilbert space $\mathcal{B}$ implies the existence of $C^{\infty}$ partitions of unity. Hence, we can extend to a $C^{k}$ map $\tilde{f}: D(A) \rightarrow \mathcal{B}$ agreeing with $f$ off a neighborhood of the relative periodic orbit $P$.

\section{Applications with Euclidean symmetry}

As an application of our results, we consider evolution equations with Euclidean $\mathbf{E}(2)$ and $\mathbf{E}(3)$ symmetry. In particular, our results are consistent with experimental, numerical and theoretical work on the meandering of spirals and give predictions for the meandering of scroll waves.

Drifts of relative equilibria for $\mathbf{E}(2)$ We consider the case of relative equilibria with isotropy $\Sigma$ in systems with overall symmetry $\Gamma=\mathbf{E}(2)$. It is convenient to define the subgroup $\Sigma_{0}=\Sigma \cap \mathbf{T}(2)$ consisting of pure translations. There are the possibilities

$$
\Sigma_{0} \cong \mathbf{1}, \quad \mathbb{Z}, \quad \mathbb{Z}^{2}, \quad \mathbb{R}, \quad \mathbb{R} \times \mathbb{Z}, \quad \mathbb{R}^{2}
$$

First suppose that the relative equilibrium has no translation symmetries, so $\Sigma_{0}=\mathbf{1}$ and $\Sigma \subset \mathbf{O}(2)$. Thus $\Sigma$ is one of the subgroups

$$
\mathbf{O}(2), \quad \mathbf{S O}(2), \quad \mathbb{D}_{k}, \quad \mathbb{Z}_{k}, \quad k \geq 1
$$

We have the following cases.

(a) $\Sigma=1\left(\mathbb{Z}_{k}\right.$ with $\left.k=1\right)$. Here, $N(\Sigma) / \Sigma=\mathbf{E}(2)$ noncompact with rank 1 . Generically, we have a rotating wave but linear translation drift occurs with codimension one.

(b) $\Sigma=\mathbb{Z}_{k}, k \geq 2$. This time, $N(\Sigma) / \Sigma=\mathbf{O}(2)$ compact with rank 1 . Linear drift is impossible. Generically, we have a rotating wave.

(c) $\Sigma=\mathbb{D}_{1} \cdot N(\Sigma) / \Sigma \cong \mathbb{R}$. Generically, we have linear translation drift parallel to the axis of the reflection symmetry.

(d) $\Sigma=\mathbb{D}_{k}, k \geq 2$, or $\mathbf{S O}(2)$ or $\mathbf{O}(2)$. No drift is possible. (The relative equilibrium is automatically an equilibrium.) 
In all cases, there is the possibility of no drift at all which occurs with codimension equal to the dimension of $N(\Sigma) / \Sigma$. Hence the codimension associated with no drift is 3 in case (a), 1 in cases (b) and (c), and 0 in case (d).

Remark 8.1 The drifts associated with nontranslation isotropy subgroups $\Sigma \subset \mathbf{O}(2)$ have implications for the meandering of spirals and target patterns observed in experiments. (We postpone references to the literature until we come to the corresponding results for the more interesting case of relative periodic solutions below.) For example, target patterns have $\mathbf{O}(2)$ symmetry and hence are stationary. Multi-armed spirals have $\mathbb{Z}_{k}$ symmetry $k \geq 2$ and are typically rotating waves. This is all in agreement with experiments. On the other hand, one-armed spirals are asymmetric and generically rotate (as is observed) but undergo translation drift with codimension one (which apparently is not observed for spirals in relative equilibrium).

There is an important issue here. At the Lie-algebraic level, a calculation shows that the codimension one translation drift marks a transition from clockwise to counterclockwise rotation. But spiral solutions have a 'preferred' direction of rotation given by the direction of the spiraling. (This might be seen as a physical explanation of the nonoccurrence of codimension one translation drift.) However, this preference, though physically self-evident, has no mathematical backing - in the framework of abstract $\mathbf{E}(2)$-equivariant evolution problems, rotation in either direction is equally likely.

Next we consider the case $\Sigma_{0} \cong \mathbb{Z}^{2}$ (a two dimensional lattice). The corresponding patterns are doubly spatially periodic and are called wallpaper patterns in the crystallographic classification and planforms in fluid dynamics. The isotropy subgroup $\Sigma$ is a semidirect product $\Sigma=H \dot{+} \mathbb{Z}^{2}$ where $H \subset \mathbf{O}(2)$ is the holohedry of the lattice ( $H=\mathbb{D}_{6}$ for the hexagonal lattice, $H=\mathbb{D}_{4}$ for the square lattice, $H=\mathbb{D}_{2}$ for the rhombic/rectangular lattice, and $H=\mathbb{Z}_{2}$ for the general lattice).

When $\Sigma=\Sigma_{0} \cong \mathbb{Z}^{2}$, we have $N(\Sigma) / \Sigma \cong H \dot{+} \mathbf{T}^{2}$ and there is generically a twofrequency toral drift. Of course, this toral drift corresponds to translations in physical space. It is of interest to observe that the rank of $N(\Sigma) / \Sigma$ exceeds the rank of $\Gamma$. This increase in the rank is not possible when $\Gamma$ is compact.

When $\Sigma$ is generated by a single (glide) reflection in addition to the translations $\mathbb{Z}^{2}$, there is generically a one-frequency translation drift parallel to the axis of reflection. Note that such solutions are rotating waves. In all other cases, $\Sigma$ contains a nontrivial rotation and a calculation shows that $N(\Sigma) / \Sigma$ is finite, hence there is no drift. The implication for applications is that doubly spatially periodic relative equilibria can drift if and only there is no rotation symmetry. Provided that there is no rotation symmetry, there is generically either a two frequency translation drift (no reflections) or a one frequency translation drift (parallel to the single axis of reflection). 
There is one special case which is viewed as a planform but not as a wallpaper pattern: namely the case $\Sigma_{0} \cong \mathbb{R} \times \mathbb{Z}$ which occurs for the rolls solution. Again genuine rolls (which have two axes of reflection symmetry) do not drift, but solutions with less symmetry generically undergo one-frequency translation drift.

The cases $\Sigma_{0}=\mathbb{Z}, \mathbb{R}$ and $\mathbb{R}^{2}$ are similar and we omit the details.

Drifts of relative periodic orbits for $\mathbf{E}(2) \quad$ Next we consider the case of relative periodic orbits in $\mathbf{E}(2)$-equivariant evolution problems. As in Section 7, there are associated subgroups $\Sigma$ signifying the symmetry of an initial condition on the relatively periodic orbit and $\widetilde{\Sigma}$ signifying the symmetry of the associated trajectory. There is also the associated cyclic subgroup $C$ whose definition we recall. Let $G=N(\Sigma) / \Sigma$ and define the projection $\pi: G \rightarrow G / G^{0}$. Then $C=\pi(\widetilde{\Sigma} / \Sigma)$ is cyclic.

It turns out that in the cases most relevant to the meandering of spirals, we have $C=1$. It follows that the expected drifts are abstractly identical to those for relative equilibria. (Of course, the abstract drift is coupled with the periodicity so the dynamics is more complicated.) We consider in turn the isotropy subgroups $\Sigma=\mathbf{1}$, $\mathbb{Z}_{k}, k \geq 2$, and $\Sigma=\mathbb{D}_{1}$.

$\Sigma=1$, one-armed spiral Our assumption that $C=1$ is equivalent to supposing that $\widetilde{\Sigma} \subset \mathbf{S E}(2)$. By Example 4.5, there is generically a one frequency toral drift, with linear unbounded drift occurring as codimension one. In the generic case, the toral drift is manifested as a physical rotation superimposed on the dynamically periodic behavior so that the overall dynamics is doubly quasiperiodic (as described in Proposition 7.1).

Winfree [20] documented the meandering or compound motion of chemical spiral waves. These motions are indeed quasiperiodic but have additional structure: whereas one would expect a two-frequency 'epicyclic' motion in dynamical phase space, the spiral appears to undergo such a motion in physical space. More precisely, there is a small-scale quasiperiodic temporal variation superimposed on a basic epicyclic planar motion. This phenomenon cannot be explained using the methods of this paper - meandering is more than drifting. Codimension one linear unbounded drift was discovered later in numerical work of Barkley [2].

It turns out that meandering in the sense of Winfree can be understood mathematically within the context of Hopf bifurcation from a relative equilibrium spiral. The first theoretical model for the meandering and linear drift of spirals is due to Barkley [3] exploiting the Euclidean invariance of the problem. Certain predictions in [3] were verified experimentally by $\mathrm{Li}$ et al [15]. The first mathematically rigorous results are due to Wulff [23]. See also [8, 11, 17, 18].

The results in the present paper give a partial explanation of the meandering of 
spirals but fail to give any insight into the codimension one transition. The details of this transition are quite remarkable. In particular, the physical obstruction to the transition discussed above for the relative equilibrium spirals is circumvented by the relatively periodic spirals. The resolution of these issues involves conceptual and technical problems which lie beyond the range of our abstract approach and we refer to $[8,11,17,18,23]$.

$\Sigma=\mathbb{Z}_{k}$, multi-armed spiral We have $N(\Sigma) / \Sigma \cong \mathbf{O}(2)$. Again, we suppose that $C=\mathbf{1}$, or equivalently that $\widetilde{\Sigma} \subset \mathbf{O}(2)$. Generically there is a one frequency rotation drift (leading to quasiperiodic dynamics) and linear drift is impossible (cf [11]). In fact, as pointed out in [11], multi-armed spirals do not meander. Rather, there is a small-scale quasiperiodic temporal variation superimposed on a rigid rotation in the plane where the speed of rotation also varies quasiperiodically. Again, the relation between drifting in phase space and meandering in physical space is beyond the abstract approach in this paper.

$\Sigma=\mathbb{D}_{1}$, spiral-antispiral bound pair The isotropy subgroup $\mathbb{D}_{1}$ corresponds to an axis of reflection symmetry joining the two spirals. We have $N(\Sigma) / \Sigma \cong \mathbb{R}$ (which is connected so automatically $C=\mathbf{1}$ ) and predict constant velocity translation drift parallel to the axis of symmetry. This result explains observations in numerical experiments of Ermakova et al [7]. By contrast, [7] find that co-rotating bound pairs of spirals (no reflection symmetry) undergo rotation drift. This behavior is also consistent with our theory, though we again have the theoretical possibility that linear unbounded drift occurs with codimension one.

The cases $C \neq 1$ are of at least theoretical interest. For example, suppose that $\Sigma=\mathbf{1}$ and $C \cong \mathbb{Z}_{2}$. This corresponds to a physical situation where a solution such as a one-armed spiral evolves in time and eventually returns to a state that is obtained from the initial state by (glide)-reflection. In that case, our prediction is for unbounded linear drift generically. Rotation drift is impossible.

Similarly, if $\Sigma=\mathbb{Z}_{k}($ so $\left.N(\Sigma) / \Sigma) \cong \mathbf{O}(2)\right)$ and $C \cong \mathbb{Z}_{2}$ then no drift is possible. Again, this corresponds physically to a periodic state that is initially a multi-armed spiral and evolves after half a period into a state that is a reflection of the initial state.

Drifts of relative periodic orbits for $\mathbf{E}(3)$ We consider relative periodic orbits for systems with $\mathbf{E}(3)$ symmetry concentrating again on the case when the cyclic subgroup $C \subset N(\Sigma) / \Sigma$ is trivial. (We have bypassed relative equilibria, but the drifts are identical since we take $C=1$.) The three-dimensional analogue of spirals 
are called scrolls and have been investigated extensively by Winfree and Strogatz, see for example [22]. A general reference is Winfree [21].

We adopt the convention that $\mathbf{O}(2)$ denotes the subgroup of $\mathbf{O}(3)$ fixing the points on a given axis in $\mathbb{R}^{3}$. (Note that this copy of $\mathbf{O}(2)$ is often denoted by $\mathbf{O}(2)^{-}$and is not contained in $\mathbf{S O}(3)$.) Subgroups $\mathbb{Z}_{k}$ and so on denote subgroups of this copy of $\mathbf{O}(2)$. In addition, $\mathbf{E}(1)$ denotes the Euclidean group corresponding to this axis and $\mathbf{E}(2)$ denotes the Euclidean group corresponding to the normal directions.

$\Sigma=\mathbf{E}(1)$, scroll wave In its simplest form, a scroll wave is a spiral extended trivially in a third direction. (So a scroll wave is a cylinder with spiral cross-section.) We have $N(\Sigma) / \Sigma \cong \mathbf{E}(2)$ and hence the results are parallel to those for one-armed spirals. The basic scroll wave is a relative equilibrium, generically a rotating wave with codimension one unbounded linear drift perpendicular to the scroll axis. The drift of relatively periodic scroll waves is similar but, coupled with the one frequency dynamics, leads to quasi-periodic and linear meandering.

$\Sigma=\mathbb{Z}_{k} \times \mathbf{E}(1)$, multi-armed scroll wave Again the results are parallel to those for multi-armed spirals.

$\Sigma=\mathbf{O}(2)$, scroll ring A scroll ring is a product of a spiral with a circle, see Winfree and Strogatz [22]. Rigid rotations and reflections of this circle make up the isotropy subgroup $\mathbf{O}(2)$. In addition, in each cross-section there is a spiral which is assumed to rotate in a periodic manner. Of course, this basic periodic dynamics does not correspond to a rigid rotation of the scroll ring, hence the scroll ring is a (relatively) periodic solution. We have $N(\Sigma) / \Sigma \cong \mathbf{E}(1)$ and hence scroll rings will generically translate parallel to their axis of rotation symmetry.

$\Sigma=\widetilde{\mathbf{S E}}(1)$, twisted scroll wave As the name suggests, a twisted scroll wave is a scroll wave with a twist along its axis. This leads to a twisting of the translation symmetry associated with untwisted scroll waves: translations along the scroll axis are coupled with rotation about the axis by an angle proportional to the translation. Observe that the twisted scroll wave is a relative equilibrium since temporal rotation about the axis is identified with axial translation. We have $N(\Sigma) / \Sigma=\mathbf{O}(2)$ consisting of rotations about the axis; thus such a pattern will generically rotate about the axis and exceptionally remain stationary.

$\Sigma=1$, twisted scroll ring and linked twisted scroll ring A twisted ring is somewhat like a Möbius band with spiral cross-section. Since spirals are oriented, there is a full $360^{\circ}$ twist instead of the usual half twist. As for the untwisted scroll ring, 
there is a rotating spiral within each cross-section. However, the temporal rotation coincides with spatial rotation so that the twisted scroll ring, like the twisted scroll wave, is a relative equilibrium.

There is an interesting contradiction here. By definition, a relative equilibrium is a steady-state coupled with drift along a group orbit. The assumption that the crosssection rotates as a spiral rotates is equivalent to assuming that the ring undergoes rotation drift. On the other hand, theory (Example 4.6) predicts linear unbounded drift generically, with rotation drift occurring with codimension one. We note that the unbounded drift is generically a corkscrew motion (translation along an axis coupled with rotation about that axis). Moreover, this axis is completely arbitrary (unrelated to any 'axis' of the scroll ring). Hence the physical manifestation of this drift will be rather complicated. Similarly, the axis of codimension one rotation drift is arbitrary. A second codimension one occurrence is that the drift remains unbounded but is a pure translation instead of a corkscrew motion. The cross-sectional behavior anticipated by [22] in defining the twisted scroll ring occurs only with codimension three! Fiedler et al [8] consider bifurcations from a twisted scroll wave translating and rotating around its axis, but such a state is still of codimension two.

Actually, Winfree and Strogatz [22] rule out the existence of single twisted scroll waves using a topological argument. Instead, they postulate the existence of a pair of linked twisted scroll rings, where the cross-sectional spiral is replaced by a spiralantispiral bound pair. This state is a relatively periodic orbit and we predict corkscrew drift (with arbitrary axis) in addition to the dynamical periodicity.

$\Sigma=\mathbb{Z}_{k}$, (linked) multi-twisted scroll ring We have $N(\Sigma) / \Sigma=\mathbf{O}(2) \times$ $\mathbf{E}(1)$. By Proposition 3.7, we have unbounded drift generically. Again, the drift is a corkscrew motion, but this time, the drift respects the axis of the scroll ring. With codimension one, the drift is either a pure translation or a pure rotation.

Drift in oscillatory systems with $S^{1}$ symmetry The context in which many of our predictions might be applicable is in oscillatory or excitable systems; reaction diffusion systems where the local dynamics is oscillatory or near-oscillatory. We now discuss some extra structure that can manifest itself in these cases as an exact or approximate $S^{1}$ symmetry in the equations.

Consider an $\mathbf{E}(2)$-equivariant reaction diffusion system on the plane. Such a system, if marginally oscillatory, is often taken to be approximated by a Complex Ginzburg-Landau equation; this is a reaction diffusion system obtained on diffusive coupling of a continuum of Hopf bifurcation normal forms. This equation possesses an $S^{1}$ symmetry with action on the range of the evolving patterns: the action of 
$(\gamma, \theta) \in \mathbf{E}(2) \times S^{1}$ on functions $u: \mathbb{R}^{2} \rightarrow \mathbb{R}^{k}$ is given by

$$
(\gamma, \theta) \cdot u(x)=\theta u\left(\gamma^{-1} x\right)
$$

where $\theta$ acts on $\mathbb{R}^{k}(k \geq 2)$. Some of the consequences of such an extra symmetry are as follows:

- Spirals are relative equilibria with 'twisted' $\mathbf{S O}(2)$ symmetry of the form $(g(\theta), \theta)$ where $g(\theta)$ is a homomorphism from $S^{1}$ to $\mathbf{E}(2)$. The connected component of the normalizer of this symmetry group contains just the symmetry group itself.

- Single and multiple arm spirals differ only by the number of 'twists' of the homomorphism $g$; i.e. they have essentially the same drifting behavior.

- Such spirals must rotate about a 'phaseless point' $x_{0} \in \mathbb{R}^{2}$, i.e. a point such that $u\left(x_{0}, t\right)$ is fixed by the action of $S^{1}$ and independent of $t$. They cannot translate.

- Isotropy and therefore predictions for the spiral-antispiral pair are unaffected.

\section{A A characterization of noncompact Lie groups}

We prove that a connected Lie group $G$ is noncompact if and only if $G$ contains a closed subgroup isomorphic to $\mathbb{R}$. The existence of such a subgroup is equivalent to the existence of an element $\xi \in L G$ such that $K(\xi) \cong \mathbb{R}$ where $K(\xi)$ is the closure of the one-parameter subgroup generated by $\xi$.

Theorem A.1 Suppose that $G$ is a connected Lie group. If $K(\xi)$ is compact for all $\xi \in L G$, then $G$ is compact.

We prove the theorem first for $G$ solvable and second for $G$ semisimple. The result for general $G$ then follows from the Levi decomposition.

Lemma A.2 Suppose that $G$ is a connected solvable Lie group and that $K(\xi)$ is compact for all $\xi \in L G$. Then $G$ is compact.

Proof We argue by induction on the dimension of $G$. The result is clear when $\operatorname{dim} G=1$. If $\operatorname{dim} G>1$, then there exists a proper connected normal subgroup $H$. The groups $H$ and $G / H$ are solvable and have lower dimension than $G$. In particular, the induction hypothesis applies immediately to $H$ so that $H$ is compact. 
We claim that $K(\eta)$ is a compact subgroup of $G / H$ for all $\eta \in L(G / H)$. It then follows by induction that $G / H$ is compact. Since $H$ and $G / H$ are compact, we conclude that $G$ is compact [4, Theorem 1.3.1].

It remains to verify the claim. Let $\pi: G \rightarrow G / H$ be projection. By the naturality of exp, we have $\pi \exp =\exp (d \pi)_{e}$. Replacing $\eta$ by an element sufficiently close to the origin, we obtain an element $\xi \in L G$ such that $(d \pi)_{e} \xi=\eta$. In particular, $\pi(\exp t \xi)=\exp t \eta$ and $\pi$ restricts to a homomorphism $p: K(\xi) \rightarrow K(\eta)$ with dense image. Since $K(\xi)$ is compact, it follows that $K(\eta)$ is compact and the claim is verified.

Remark A.3 Under the hypotheses of Lemma A.2, $G$ is a torus. (One way to see this is to use the fact that the irreducible (complex) representations of a solvable group are one dimensional. The only compact groups with this property are tori.)

Lemma A.4 Suppose that $G$ is a connected semisimple matrix group. If $K(\xi)$ is compact for all $\xi \in L G$, then $G$ is compact.

Proof Since $G$ is a matrix group, the condition that $K(\xi)$ is always compact is equivalent to the condition that each $\xi \in L G$ is semisimple with purely imaginary eigenvalues. Consider the symmetric bilinear map $Q(\xi, \eta)=-\operatorname{tr} \xi \eta$. The eigenvalues of $\xi$ are purely imaginary, so $Q(\xi, \xi) \geq 0$ for all $\xi \in L G$. If $Q(\xi, \xi)=0$, then $\xi$ is nilpotent. By assumption, each $\xi$ in $L G$ is semisimple, so $\xi=0$. Hence $Q$ defines an inner product on $L G$. Moreover, this inner product is preserved by the adjoint action of $G: Q\left(\operatorname{Ad}_{g} \xi, \operatorname{Ad}_{g} \eta\right)=Q(\xi, \eta)$ for all $g \in G, \xi, \eta \in L G$.

Let $\mathbf{O}(L G)$ denote the orthogonal subgroup of $\mathbf{G L}(L G)$ corresponding to this inner product. Then $\mathbf{O}(L G)$ is compact and $\operatorname{Ad}(G) \subset \mathbf{O}(L G)$. By [19, Theorem 3.10.8], $\operatorname{Ad}(G)$ is a closed subgroup of $\mathbf{G L}(L G)$. Hence $\operatorname{Ad}(G)$ is compact. Finally we appeal to the semisimplicity of $G$. Indeed, the group $G$ is a covering of $\operatorname{Ad}(G)$ (since ker Ad coincides with the center of $G$ which is discrete). It follows from Weyl's Theorem [19, Theorem 4.11.6] that $G$ is compact.

Lemma A.5 Suppose that $G$ is a connected semisimple Lie group. If $K(\xi)$ is compact for all $\xi \in L G$, then $G$ is compact.

Proof Let $G^{\prime}=G / Z(G)$ where $Z(G)$ is the center of $G$. Then $G^{\prime}$ has no center and it follows that $G^{\prime}$ is a matrix group (the isomorphism being given by $\operatorname{Ad}: G^{\prime} \rightarrow$ $\left.\operatorname{GL}\left(L G^{\prime}\right)\right)$. The groups $G$ and $G^{\prime}$ have the same Lie algebra $L G$. If $\xi \in L G$, we write $K_{G}(\xi)$ to denote the copy of $K(\xi)$ contained in $G$. It follows from naturality of exp that the projection $\pi: G \rightarrow G^{\prime}$ restricts to an isomorphism of the corresponding oneparameter subgroups in $G$ and $G^{\prime}$ and defines a smooth homomorphism $p: K_{G}(\xi) \rightarrow$ 
$K_{G^{\prime}}(\xi)$ with dense image. Since $K_{G}(\xi)$ is compact, it follows that $K_{G^{\prime}}(\xi)$ is compact. It now follows from Lemma A.4 that the semisimple matrix group $G^{\prime}$ is compact. Applying Weyl's Theorem, we deduce that $G$ is compact.

Proof of Theorem A.1 The Levi decomposition for Lie groups [19, Theorem 3.18.13] states that $G=Q M$ where $Q$ is solvable and $M$ is semisimple. At the Lie algebra level, we have the direct sum of vector spaces $L G=L Q \oplus L M$ where $L Q$ is solvable and $L M$ is semisimple. By Lemmas A.2 and A.5, $Q$ and $M$ are compact. The multiplication $Q \times M \mapsto G$ is surjective so $G$ is compact.

Acknowledgments We are grateful to Mike Field and Valentin Krinsky for several helpful discussions.

\section{References}

[1] R. Abraham, J. E. Marsden and T. Ratiu. Manifolds, Tensor Analysis, and Applications, Addison-Wesley, Reading, Mass., 1983.

[2] D. Barkley. A model for fast computer-simulation of waves in excitable media. Physica D 49 (1991) 61-70.

[3] D. Barkley. Euclidean symmetry and the dynamics of rotating spiral waves. Phys. Rev. Lett. 72 (1994) 165-167.

[4] G. E. Bredon. Introduction to Compact Transformation Groups. Pure and Appl. Math. 46 (New York: Acad. Press, 1972).

[5] T. Bröcker and T. tom Dieck. Representations of Compact Lie Groups. Grad. Texts in Math. 98, Springer, New York, 1985.

[6] R. W. Carter, G. Segal and I. G. MacDonald. Lectures on Lie Groups and Lie Algebras. London Math. Soc. Student Texts 32, Camb. Univ. Press, 1995.

[7] E. A. Ermakova, A. M. Pertsov and E. E. Shnol. On the interaction of vortices in two-dimensional active media. Physica D 40 (1989) 185-190.

[8] B. Fiedler, B. Sandstede, A. Scheel and C. Wulff. Bifurcation from relative equilibria of noncompact group actions: skew products, meanders and drifts. Documenta Math. 1 (1996) 479-505.

[9] M. J. Field. Equivariant dynamical systems. Trans. Amer. Math. Soc. 259 (1980) 185-205. 
[10] M. J. Field. Local structure for equivariant dynamics, in Singularity Theory and its Applications Part II, (M. Roberts, I. Stewart, eds.), Lecture Notes in Math. 1463, Springer, Berlin, 1991, 142-166.

[11] M. Golubitsky, V. G. LeBlanc and I. Melbourne. Meandering of the spiral tip, an alternative approach. To appear in J. Nonlin. Sci.

[12] D. Henry. Geometric Theory of Semilinear Parabolic Equations. Lecture Notes in Math. 840, Springer, Berlin, 1981.

[13] B. R. Hunt, T. Sauer and J. A. Yorke. Prevalence, a translation-invariant 'almost every' on infinite-dimensional spaces, Bull. Amer. Math. Soc. 27 (1992) 217-238.

[14] M. Krupa. Bifurcations of relative equilibria. SIAM J. Math. Anal. 21 (1990) 1453-1486.

[15] G. Li, Q. Ouyang, V. Petrov and H. L. Swinney. Transition from simple rotating chemical spirals to meandering and traveling spirals. Phys. Rev. Lett. 77 (1996) 2105-2108.

[16] S. Lojasiewicz. Ensembles Semi-Analytiques. IHES Lecture Notes, 1965.

[17] B. Sandstede, A. Scheel and C. Wulff. Center-manifold reduction for spiral waves. C. R. Acad. Sci. To appear.

[18] B. Sandstede, A. Scheel and C. Wulff. Dynamics of spiral waves in unbounded domains using center-manifold reductions. Preprint.

[19] V. S. Varadarajan. Lie Groups, Lie algebras, and their Representations. Grad. Texts in Math. 102, Springer, New York, 1984.

[20] A. T. Winfree. Scroll-shaped waves of chemical activity in three dimensions, Science 181 (1973) 937-939.

[21] A. T. Winfree. When Time Breaks Down. Princeton Univ. Press, Princeton NJ, 1987.

[22] A. T. Winfree and S. H. Strogatz. Organizing centres for three-dimensional chemical waves. Nature 311 (1984) 611-615.

[23] C. Wulff. Theory of Meandering and Drifting Spiral Waves in Reaction-Diffusion Systems. Freie Universität Berlin, Thesis, 1996. 